\title{
Preschoolers' Use of Superordinate Relations in Classification and Language
}

\author{
Sandra Waxman \\ Rochel Gelman \\ University of Pennsylvania
}

\begin{abstract}
Preschoolers' difficulty in accessing superordinate relations in classification contrasts sharply with their facility in accessing superordinate relations in language use. We consider two hypotheses regarding this discrepancy. First, certain aspects of classification tasks may obscure superordinate relations. In free classification tasks, the open-ended instructions leave the choice among possible organization schemes unconstrained, allowing for virtually any grouping (e.g., thematic, idiosyncratic), not necessarily a taxonomic grouping. Second, children's facility with superordinate relations in language may be due to a language-specific constraint in development: Children may interpret novel labels as referring to taxonomic (as opposed to thematic or idiosyncratic) relations. In Experiment I, we used 'clues' to focus preschoolers' attention on superordinate relations. Clues were (1) superordinate category labels (Label condition); (2) sets of typical category instances (Instance condition); or (3) typical instances with instructions to consider the instances as a group (Group condition). Four-year-olds classified successfully in all conditions. Three-year-olds classified well with labels, but not with instances; their performance in the Group condition was intermediate. In Experiment II, we focus on the role of labels in superordinate classification. If labels highlight taxonomic relations, then a set of category instances paired with a novel label should serve as an especially effective taxonomic clue. With the introduction of a novel word, 3-year-olds classified as successfully as children provided with known English labels. The powerful relation between language and classification is discussed.
\end{abstract}

Classification is a powerful cognitive process, allowing us to organize a great deal of information efficiently and flexibly. In forming classes, we highlight important similarities among objects and events, treating very different items as

Support for the research came from NSF grant BNS-8004881 to RG. Parts of this paper were presented in a paper by SW entitled Superordinate Classification in Preschool Children at the conference of the Society for Research in Child Development, April, 1983, Detroit. RG helped finish the manuscript while she was a fellow at the Center for Advanced Study in the Behavioral Sciences and supported by funds from Alfred P. Sloan and Spencer Foundation as well as NIHHCD Senior Fellowship \# F33HD06623. We are grateful to M. Callanan, L. Gleitman, E. Meck, R. Ostrin, H. Schmidt, E. Shipley, and E. Spelke for helpful comments on earlier versions of this paper. Special thanks to J. Sabini for suggestions that led to Experiment II. 
equivalent for certain purposes. Of course, any particular item can be considered a member of many different classes. For example, an apple belongs to the classes 'apple', 'fruit', 'food', 'physical object', etc. These classes are hierarchically organized; they honor inclusion relations. Taxonomic classification systems such as these have a long philosophic history and have been adapted to studies of human concept structure (see Smith \& Medin, 1981). However, not all classifications are taxonomic. We also can use thematic relations (e.g., grouping an apple with a knife) and idiosyncratic or ad-hoc relations (e.g., grouping an apple with a snake to form the class 'Biblical symbols'). Because classification is flexible, we can exploit different relations among objects, depending upon the task at hand. Preschoolers' appreciation of thematic and idiosyncratic relations has been clearly demonstrated (Inhelder \& Piaget, 1964; Nelson, 1978; Mandler, 1983). Their appreciation of taxonomic relations is less clcar.

Researchers interested in young children's appreciation of taxonomic relations have based much of their work on two measures. First, they observe children's object classification, on the assumption that children's spontaneous groupings reflect their underlying conceptual organization (Clark, 1983). Second, they observe children as they begin to talk about objects, on the assumption that children's speech reflects their underlying conceptual organization. At the basic level (e.g., dogs, apples, shirts), these measures converge on one of the most robust findings in the developmental literature. Preschool children readily classify (Rosch, Mervis, Gay, Boyes-Braem, \& Johnson, 1976; Sugarman, 1983) and label items (Anglin, 1979) at the basic level. Preschoolers' appreciation of taxonomic relations at superordinate levels (e.g., animals, food, clothing) is more controversial. Here, the two experimental measures do not converge. Preschoolers' difficulty in using superordinate relations in classification stands in sharp contrast to their facility in language. This contrast between children's access to superordinate relations in classification and language serves as the focus of this paper.

Preschoolers' difficulty in classifying objects at superordinate levels is well documented (see Gelman \& Baillargeon, 1983; Markman \& Callanan, 1983 for reviews). In most superordinate classification tasks, young children prefer to sort on the basis of thematic or idiosyncratic, rather than taxonomic, relations. However, when performance demands are minimized, preschoolers are sometimes able to use superordinate relations in classification. For example, Markman, Cox, and Machida (1981) demonstrated that when competing tendencies are reduced by having preschoolers sort objects into bags rather than on an open surface (on a table), children are better able to classify at superordinate levels. Even under these favorable conditlons, however, superordinate classification was not easily accomplished by these young children; only $40 \%$ of the items in their task were classified on the basis of superordinate relations. In sum, although young children can sometimes use superordinate relations in classifica- 
tion, this is accomplished with effort-either on the part of the experimenter designing the task or on the child executing it.

When considering the young child's use of language, a very different picture emerges. Here, children are quite sensitive to superordinate relations and, in fact, seem to use them naturally and spontaneously. For example, Rescorla (1981) has shown that overextensions are most often within superordinate category boundaries. Toddlers overextend the word $d o g$ to refer to other animals, but not to items outside this category. Preschoolers' sensitivity to superordinate relations is also evident in their 'definitions'. When asked to 'define' superordinate terms (e.g., animals), they readily list a series of category members (Anglin, 1977). Further, Keil (1979) found that, like adults', 3-year-old children's judgments reflect an implicit hierarchial organization for ontological knowledge. Children maintained that it is silly to say that inanimate objects (c.g., cars) arc "sorry," but said that animate objects could be so described. (Also, see Landau \& Gleitman, 1985, for further evidence on this topic.)

If preschoolers appreciate superordinate relations and use them naturally in language, how can we account for their difficulty in most classification tasks? We consider two hypotheses. First, there may be some particular characteristic of classification tasks that obscures the superordinate relations. Second, there may be some particular characteristic of word learning which makes these relations especially salient in a word-learning context. These hypotheses are not mutually exclusive. Together they may account for the discrepancy in children's performance.

Aspects of classification tasks that may obscure superordinate relations: The first possibility, that preschoolers' difficulty stems, at least in part, from something inherent in classification tasks themselves, has been raised by other researchers (e.g., Markman \& Callanan, 1983). In view of their results, we designed a very simple classification procedure, minimizing extraneous performance demands. Children formed only one category at a time; they classified one stimulus at a time; and they used bins to help organize their classifications. We adopted this simple procedure to focus on another aspect of the classification task-its openendedness.

In the standard free-classification task (Inhelder \& Piaget, 1964), children are presented with a set of materials (objects or pictures) and instructed simply to "put the things which belong together together," leaving it to the child to discover and impose an organizational scheme. ${ }^{1}$ These open-ended instructions

\footnotetext{
I Piaget had a principled reason for keeping the instructions vague: He argued that true classification involved the coordination of an intensional and an extensional definition of a class. Thus, by providing children with tasks requiring that they find the intension and then the extensions of a class, he hoped to detect the point at which these two aspects of true classification became coordinated. However, there is another serious consequence to using these instructions: Because they are open-
} 
allow for virtually any grouping of the materials; they do not call specifically for a taxonomic classification. Rather, children are free to impose whatever organizational scheme they prefer. Thus, failure to use superordinate relations in classification may reflect the fact that it is not clear to children that they are to sort taxonomically. To be sure, there is a difference here between children and adults. In an open-ended task of this kind, adults almost invariably respond on the basis of taxonomic relations while young children do not. Still, the question is whether this is a difference in preference only (an interesting fact in its own right) or a categorical difference in the conceptual organization of children and adults.

Since children of this age find other relations (thematic, idiosyncratic) more salient in classification (Smiley \& Brown, 1979), we provided children with clues, specifically designed to highlight superordinate category relations. We predicted that these clues would clarify the task and facilitate superordinate level classification. ${ }^{2}$ In the first experiment, we used three kinds of clues to highlight the intended superordinate categories. In the Label condition, children were given the superordinate category label (e.g., animal) as their clue. In the Instance condition, we indicated the intended superordinate category by reference to a set of its typical members (e.g., dog, horse, duck). Based on an earlier finding (Waxman, 1983) that preschoolers tend to consider each instance mentioned as a separate, freestanding category, rather than generalizing from those instances to the common higher order group (e.g., animals), we included a third experimental condition. In the Group condition, we explicitly encouraged the children to think of the instances together, as members of a common group. In sum, children in the Label condition had the superordinate category term presented to them directly. Children in the Instance condition had to generate the intended superordinate category themselves from a set of instances. Children in the Group condition were provided with instances and the explicit encouragement to generate a higher order category.

If preschoolers' difficulty in superordinate classification reflects, at least in part, the open-endedness of the instructions, then clues should facilitate classification. Although this would provide evidence for our first hypothesis, it raises an intriguing question. Why would children need clues to focus on superordinate

ended, one should not interpret children's failure to impose a taxonomic classification scheme as an indication that they lack a classification structure.

In addition, there is philosophical bias inherent in the design of the free-classification task. It presupposes that concepts do have intensional definitions. However, see Kripke's (1972) arguments on purely extensional theories of concepts.

${ }^{2}$ Notice that what one chooses to use as a clue is not independent of one's view of the structure of concepts. For example, classical theory would predict that definitional clues would be most useful, while prototype theories would suggest that prototypical category members would be most useful. The serious question of how to best characterize the structure of concepts is beyond the scope of this paper. See Smith and Medin (1981) for a review of this topic. 
relations in classification but not in word learning? Surely, the task of word learning is at least as open-ended or unconstrained as that of object classification. Consider Quine's (1960) induction problem: When an object is labeled for a child (for example, when a caretaker points to an object saying, "That's a cat."), how is the child to know that the label refers to the cat itself (and not the cat and its milk bowl, or the cat's tail, etc.) and all other cats as well? How is the child to know that such a term refers to a specific object and to a taxonomic class, but not to several other possible relations? This brings us to our second hypothesis: that there is something special about the task of word learning that makes taxonomic relations especially salient.

Aspects of word learning that may highlight taxonomic relations: There is evidence that very young children are sensitive to linguistic cues and use them to help determine the meaning of ncw words. For example, children use linguistic form class to decide whether a novel word refers to an action, an object, or a substance (Brown, 1957). They also know that nouns can refer to individual items (proper nouns) as well as to classes of items (common nouns), and they appreciate the linguistic cues that make this distinction (Katz, Baker, \& MacNamara, 1974; Gelman \& Taylor, 1984). Finally, Markman and Hutchinson (1984) propose that young children honor an implicit bias in word learning, assuming that novel nouns refer to taxonomic, rather than thematic, relations among objects.

In Markman and Hutchinson's (1984) triad tasks, the experimenter pointed to a picture of a 'standard' item (e.g., a dog) and asked children to select one of the two 'target' items. One target was thematically related to the standard (e.g., dog food); the other was taxonomically related to the standard (e.g., a cat). Markman and Hutchinson report that when the experimenter pointed to the standard and asked children to "find another one," they tended to choose the thematically related target. However, when the experimenter labeled the target with a nonsense word and asked children to "find another dax," they tended to choose the taxonomically related target. Based on these results, Markman and Hutchinson claimed that the introduction of the novel label caused children to override their thematic bias, shifting their attention to the taxonomic relations among the items. They argue that this taxonomic bias in word learning operates at both the basic (for 2- and 3-year-old children) and superordinate (for 4- and 5-year-old children) levels.

While this proposed constraint regarding nouns and category relations would be particularly useful to young children in their language and conceptual development, the evidence for this constraint is not conclusive. There are several issues, both methodological and theoretical, which remain unresolved. First, since Markman and Hutchinson (1984) included only one alternative to the preferred, thematically related target item, and since that alternative was always a taxonomically related target item, we cannot unambiguously determine what role the novel label played in their task. While it is possible that the label induced 
children to choose taxonomically, as Markman and Hutchinson suggest, an alternative interpretation is also possible. Perhaps introducing an unusual label alerted children to something unusual about the task and made their usual choice for the thematically related item less likely. The label may have biased the children in favor of the taxonomic choice; but it also may have biased them against the thematic choice. The first interpretation argues for a specific and powerful constraint on word meaning. The second interpretation argues for a more general effect brought about by demand characteristics.

To resolve this ambiguity, it is necessary to use methods extending beyond triad tasks or 'forced choice' procedures in which children make only a single decision in selecting a target itcm. We chose a classification task in which the child's task was to establish and maintain taxonomic organizational schemes across a series of choices. This more stringent measure allowed us to assess the hypothesis that labels consistently highlight taxonomic relations among items for young children.

Second, Markman and Hutchinson (1984) argue that " . . . labels cause children to search for category relations..." and that this powerful constraint ". . . tremendously simplifies the problem of language learning" (p. 23). We agree that such a bias would be most useful, but we interpret the data more cautiously. For example, at the superordinate level, the 4- and 5-year-old children who heard the novel would chose the taxonomically related item on but $65 \%$ of their trials. This is significantly higher than chance performance (50\%), but we would expect to have seen a more dramatic effect as a consequence of a substantive constraint on language.

Perhaps most important, there is an important theoretical question regarding the role of labels in classification. Even in the absence of a novel label, young children establish basic level classes. They find it considerably more difficult to establish higher order superordinate-level classes (e.g., Rosch et al., 1976). If there is an implicit bias to interpret novel labels taxonomically, it would be most beneficial to young children at superordinate levels where they have difficulty establishing taxonomic relations. Recall that Markman and Hutchinson (1984) examine the effect of novel labels at the basic level only for 2- and 3-year-old children but have not examined their effect at superordinate levels in children younger than 4 or 5 years of age. If single nouns draw attention to taxonomic relations among objects, and if this bias is present early in development, then novel labels should serve as especially effective clues for very young children in superordinate-level classification.

We test this hypothesis in Experiment II, by introducing a new experimental clue - a Japanese label - in our superordinate classification task. Children in Japanese Label condition heard a novel (Japanese) label in conjunction with the set of instances. In all other ways the procedure used in the Japanese Label condition was identical to that in the Instance condition. By comparing performance in the Japanase Label condition to that in the other clue conditions, we 
observe the effect of labels in highlighting superordinate relations among objects.

\section{EXPERIMENT I}

\section{Method}

Subjects. Thirty-six preschoolers, enrolled in area preschool programs serving a racially mixed, middle-class population, served as subjects. Two age groups were tested: 183 -year-olds (mean age $=3.7$ ) and 18 4-year-olds (mean age $=4.5$ ). Approximately equal numbers of boys and girls were included in each age group.

Stimuli. Thirty-one drawings were selected from Snodgrass and Vanderwart's (1980) standardized line drawings. ${ }^{3}$ (See Table 1 for a complete list of stimuli.) These were magnified to twice their original size, mounted on $11 \times 15$ $\mathrm{cm}$ cards, and laminated for protection. Ten of these drawings were used only in the familiarization period. The remaining 21 were used only in the experimental task itself. These included 7 cards from each of the 3 target superordinate categories under investigation-animals, clothing, and food.

Procedure. All children were tested individually in a quiet room within their preschools. They were randomly assigned to one of three experimental clue conditions (Label, Instance, Group). All children participated in a brief familiarization period. Next, children in all conditions classified the experimental stimuli three times, selecting a different category (animals, clothing, or food) on each trial. Order of category presentation was completely counterbalanced. Between trials, the drawings were shuffled and presented again in random order. Finally, all children participated in a free-classification posttest.

Familiarization Period. All children participated in an identical familiarization period. The purposes of this period were (a) to make, sure that each child could identify all of the stimuli used in the experiment and (b) to familiarize them with the experimental procedure, including the two sorting bins and the puppets. First, each child was asked to name the drawings. The experimenter named those that the child was unable to identify. Next, the experimenter introduced the child to a hand puppet and said, "Do you think this puppet likes everything? No, he

\footnotetext{
${ }^{3}$ In order to assess young children's judgments of item typicality, we used a sample of Snodgrass and Vanderwart's (1980) standardized line drawings. (These were magnified to three times their size.) We made three posters, one for each category (animals, clothing, food). Each poster measured $45 \mathrm{~cm} \times 45 \mathrm{~cm}$ and contained 15 drawings from its target category as well as 10 distractors. Each drawing was approximately $8 \times 8 \mathrm{~cm}$. Ten 3 - and 4-year-old children were asked to point to the animals, clothes, or food on the appropriate poster. Only those items selected by all 10 children were used in these experiments.
} 
Table 1. List of Selected Stimuli

\begin{tabular}{|c|c|c|c|c|}
\hline & \multicolumn{2}{|c|}{ Clues } & \multicolumn{2}{|c|}{ Items } \\
\hline & Labels & Instances & Target Items & Distractors \\
\hline Familiarization Period & Furniture & $\begin{array}{l}\text { chair } \\
\text { dresser } \\
\text { stool }\end{array}$ & $\begin{array}{c}\text { table } \\
\text { desk } \\
\text { bed } \\
\text { couch }\end{array}$ & $\begin{array}{l}\text { sandwich } \\
\text { barn } \\
\text { tomato }\end{array}$ \\
\hline \multirow[t]{3}{*}{ Experimental Tasks } & Animal & $\begin{array}{c}\text { dog } \\
\text { horse } \\
\text { duck }\end{array}$ & $\begin{array}{c}\text { cow } \\
\text { bunny } \\
\text { kitten } \\
\text { bird }\end{array}$ & \\
\hline & Clothes & $\begin{array}{l}\text { pants } \\
\text { jacket } \\
\text { blouse }\end{array}$ & $\begin{array}{c}\text { dress } \\
\text { shirt } \\
\text { skirt } \\
\text { sweatshirt }\end{array}$ & \\
\hline & Food & $\begin{array}{l}\text { apple } \\
\text { celery } \\
\text { grapes }\end{array}$ & $\begin{array}{c}\text { corn } \\
\text { carrot } \\
\text { lemon } \\
\text { banana }\end{array}$ & \\
\hline
\end{tabular}

only likes some things. I'll tell you what he likes. He only likes things called 'furniture'. Do you know what furniture is? It's things like a bed, or a desk, or a chair." As she mentioned it, she placed a drawing of each item in a bin in front of the child. The experimenter then asked the child to find other things that the puppet would like, explaining that the things the puppet liked would go in one plastic bin, and that things he did not like would go in the other. Four additional drawings of furniture and three distractors were then sorted by the child with feedback from the experimenter. The experimenter presented the drawings one at a time, in random order, naming each at the basic level (e.g., couch) as she showed it to the child. Neither the drawings nor the category (furniture) used in the training trial were included in the experiment proper.

Notice that in the familiarization period, the experimenter provided both the superordinate label ('furniture') and three instances for the child. In the experiment proper, only one of these was provided as a clue (see below). In the Label condition, the label was presented, but the instances were not; in the Instance and Group conditions, the instances were presented, but the label was not.

Experimental Session. All children were now introduced to three new puppets which the experimenter described as ". . . very picky." Children in the Label, Instance, and Group conditions received different types of clues for classification. In the Label condition, the superordinate category labels served as clues. No instances of the category were provided. Holding up the first puppet, for example, the experimenter said "This puppet only likes animals." Children 
were then asked to choose other things the puppet would like. They were instructed to put the pictures of things for the puppet into one bin and everything else into another. When the children had completed sorting the stimuli for this puppet, the experimenter introduced the second puppet and said, for example, "This puppet only likes clothes." Again, the child went through the set of stimuli, this time selecting things for the second puppet. This procedure was repeated once more for the third category.

For children in the Instance condition, the experimenter began by showing the child three instances of each superordinate category, without mentioning the superordinate label. In this condition, the experimenter said, for example, "This puppet only likes things like a dog, or a horse, or a duck, and other things like that." These instances were left, face down, in the target bin as the child sorted the remaining stimuli. As in the Label condition, children were asked to put the things the puppet would want into one basket and everything else into another.

Instructions in the Group condition differed from those in the Instance conditions in only one respect. After the three instances for each superordinate category were presented, the experimenter said, " . . . Look, those make a really good group. They really go together well, don't they? How come they make such a good group. . . ?' In all other ways, children in the Group and Instance conditions were treated identically. ${ }^{4}$

Posttest: Free Classification. In order to provide a rough point of comparison between performance in our modified task and the standard free-classification procedure, each child's session ended with a free-classification trial. Following Inhelder and Piaget (1964), the experimenter handed the child the entire set of 12 drawings and said, ". . . Put the things that belong together in the same pile. . " The drawings were exactly those used in the experimental session.

\section{Scoring}

Experimental Task. A score for each classification trial (animals, clothing, food) was determined by counting the number of items the child placed correctly on that trial. For example, when classifying the animals, if a child placed all four drawings of animals in the target bin, and all eight remaining drawings in the other bin, the child would receive a score of 12 . If a child placed all four drawings of animals (correctly) as well as thrce other drawings (incorrectly) in the target bin, and the remaining five drawings (correctly) in the other bin, the child would receive a score of 9 . The probability of placing any item correctly is $50 \%$. Chance performance would yield a score of 6 on each trial.

In addition, we analyzed children's classifications using the scoring system

4 The experimenter made these general comments in an effort to encourage children to consider the instances together. Although the children generally agreed that the instances did 'go together', they did not spontaneously answer why they made such a good group. 
established by Markman et al. (1981). ${ }^{5}$ The two scoring systems revealed the same effects, with only one exception. That exception is reported below. For all other effects, the results are based on the first scoring system (scores ranging from 0 to 12).

Posttest. Children's free classifications were rated as follows, using Inhelder and Piaget's (1964) criteria: Type (i) children played with the drawings, or placed them randomly; Type (ii) children formed thematic relations or built patterns; Type (iii) children began to classify taxonomically, but produced errors of ommission or commission; Type (iv) children generated consistent and exhaustive taxonomic sorts. Two independent raters scored all of the sorts and agreed on $92 \%$ of the cases. Disagreements were resolved by discussion.

\section{Results}

In general, the children performed well in this classification task. While 4-yearolds performed at a consistently high level in all three experimental conditions, 3-year-olds' performance was sensitive to the differences among the various clues (see Figure 1). Since the only difference among the experimental conditions was in the type of information provided as clue, differences among the experimental groups reflect the role of the clues themselves, rather than our relaxation of performance demands.

The data were submitted to a three-way (Age $\times$ Clue-type $\times$ Category) analysis of variance. Four-year-olds performed significantly better than 3-yearolds, $F(1,30)=16.53, p<.0005$. The main effect for Clue-type indicates that performance was best in the Label condition, poorest in the Instance condition, and intermediate in the Group condition, $F(2,30)=4.75, p<.02$. As expected, there was no effect for Category; children's performance was comparable when classifying the animals, clothing, and food.

The Age $\times$ Clue-type interaction was significant, $F(2,30)=3.25, p<.05$ (see Figure 1). While 4-year-olds performed well in all clue conditions, 3-yearolds' performance varied with clue-type. Mean scores for the 4-year-olds in the Label, Instance, and Group conditions were 11.61, 11.39, and 11.44, respectively. Three-year-olds sorted successfully in the Label condition (mean score $=$ 11.39), but had more difficulty in the Instance condition (mean score $=9.00$ ). Performance in the Group condition fell between these two extremes (mean score $=9.72$ ). Tukey pair-wise comparisons of the 3-year-olds' data revealed that performance in the Label condition was significantly more accurate than performance in either the Instance or the Group condition $(p<.05)$; the difference

\footnotetext{
5 Following Markman et al. (1981), a score for each classification trial was determined by subtracting the number of incorrectly classified drawings from those correctly included in each category. In this system, the maximum possible score for any classification is 4 (4 correct drawings per category); the minimum score is held at 0 .
} 


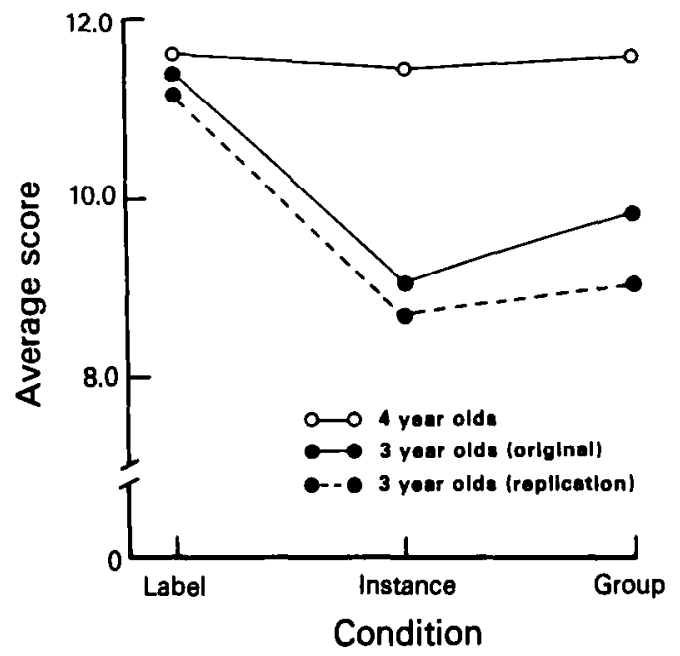

Figure 1. Experiment I. Mean scores for 3- and 4-year-old children in the Label, Instance, and Group Clue conditions

between the Instance and Group conditions was in the expected direction, ( $p$ $<.07)$.

These results were based on 6 subjects in each age group per condition. As a check on the reliability of these results, we ran 18 additional 3-year-olds, drawing from the same population as above. The mean scores were $11.25,8.81$, and 9.33 for the Label, Instance, and Group conditions, respectively (see Figure 1). This replicated the pattern reported above. Since there were no significant differences between these two samples of 3-year-olds, their results were combined and submitted to a two-way (Clue Type $\times$ Category) ANOVA.

The main effect for Clue-type, $F(2,30)=4.52, p<.02$, replicated our previous finding: Children classified best in the Label condition and had most difficulty in the Instance condition. Performance in the Group condition was intermediate. Tukey pair-wise comparisons indicated that children performed significantly better in the Label than in the Instance condition $(p<.05)$, but that neither of these differed reliably from the intermediate Group condition.

There also was a main effect for Category, $F(2,60)=4.01, p<.02$. Threeyear old children classified best in the food category (mean score $=10.19$ ), next in the clothing category (mean score $=9.92$ ), and least well in the animal category (mean score $=9.28$ ). Only the difference between the food and animal categories was reliable $(p<.05)$. This effect was not obtained in the second analysis, based on Markman et al.'s (1981) scoring system.

Performance on the free-classification posttest task replicated the pattern of results reported by Inhelder and Piaget (1964). Although the stimuli were identical to those used in the experimental session, children had much more difficulty 
in free classification. Only 6 (17\%) 3-year-olds sorted consistently and exhaustively. Despite 4-year-olds' success in all conditions in the preceding experimental task, only $9(50 \%)$ of them were able to maintain consistent superordinate classification schemes in the free classification task.

\section{Discussion}

Four-year-olds' successful classification in all clue condition provides support for our first hypothesis: that difficulty using superordinate relations in classification tasks stems, at least in part, from the open-endedness of the task. When their task is clear to them, 4-year-olds impose superordinate classification schemes consistently and exhaustively. With clues presented in the context of a simplified procedure, these children readily recognized the superordinate-level taxonomic relations and used them to classify the materials categorically. Without clues, in the free-classification pottest, half of the 4-year-olds failed to produce consistent superordinate sorts, despite the fact that the categories and stimuli were identical to those used in the preceding experimental tasks. Clues, designed to limit the number of possible organizational schemes, facilitated superordinate-level classification for the 4-year-old children.

Unlike the 4-year-olds, the 3-year-olds' ability to classify at the superordinate level was directly related to the type of information provided as a clue. With category labels, 3-year-olds succeeded in classifying at the superordinate level. Previous work (Kobasigawa \& Middleton, 1972) has shown that labels facilitate classification in school-aged children. Our work extends this result to preschool children. The fact that preschoolers were able to use superordinate labels reveals two important points: First, although superordinate terms are not often used by preschool children, and are seldom introduced by their parents (Shipley, Kuhn, \& Madden, 1983; Callanan, 1982), these children understand their meaning. Second, preschoolers readily use their knowledge of superordinate classes to impose a consistent taxonomic organization.

Three-year-olds' performance in the Instance condition replicated our earlier finding that preschool children often have difficulty generating higher order categories from a set of instances (Waxman, 1983). This finding is somewhat surprising. Young children can generalize from instances to basic-level taxonomic classes (Kendler \& Guenther, 1980; Mervis \& Pani, 1980; Horton \& Markman, 1980). They also offer typical category members when asked to define superordinate-level categories (Anglin, 1977). What could account for the younger children's relative difficulty using typical instances as clues in our superordinate-level classification task? The children may not have understood that they were to generalize from the three instances to the higher order superordinate-level class. (After all, the puppets were described as 'picky' and if the experimenter had meant include the entire class of animals, for example, she probably would have said so.) Perhaps very young children need some form of explicit instruction, encouraging them to form the higher order category and use 
it as a basis for sorting. However, even when 3-year-olds were explicitly encouraged to consider the instances together as a group (Group condition), their performance was statistically indistinguishable from that in the Instance condition. (Although 3-year-olds' performance in the Group condition did not differ reliably from either the Instance or Label conditions, notice that the mean score for the Group condition is closer to the mean score for the Instance condition than it is to the mean score for the Label condition.) Apparently, even these explicit instructions did not sufficiently highlight the taxonomic relations among the instances for the 3-year-old children. Between the ages of 3 and 4, children develop a greater facility at inducing superordinate categories from instances.

Thus, although 4-year-olds classified well in all clue conditions, the 3-yearolds' performance varied considerably under each of the three experimental clue conditions. Pcrhaps the variation in the younger children's performance simply reflects the degree to which each of the experimental conditions was related to the familiarization period. Since children were provided with both the superordinate label and the instances as clues in the familiarization period, this is unlikely. In the subsequent experimental sessions, the labels alone (with no instances) proved to be a successful clue; the instances alone (with no labels) did not. Further, notice that the instructions in the Group condition differed most from those in the familiarization period, yet performance in this condition fell between performance in the Label and Instance conditions.

Overall, the superordinate label was the most effective clue in eliciting consistent taxonomic classification. This is just the kind of result predicted by Markman and Hutchinson's (1984) view that labels are powerful taxonomic clues. However, one might argue that success in our Label condition may have been due to semantic associations between superordinate labels and category members as opposed to the process of labeling, per se. It may be that children in the Label condition relied solely on well-established semantic associations between the superordinate labels and individual category members. Children learn, for example, that a horse is a kind of animal, and that an apple is a kind of food. On the other hand, it may be that the process of labeling, per se, calls attention to taxonomic relations among objects. Because we used well-known labels in this experiment, the effects of labeling and of semantic associations were confounded. Therefore, these data cannot provide an answer to this important question. Experiment II was designed to disentangle these two alternatives.

\section{EXPERIMENT II}

In this experiment, we test the hypothesis that children as young as 3 years of age honor an implicit taxonomic bias in word learning. We predict that a novel label will highlight the taxonomic relations among instances and will serve as an especially effective clue in superordinate classification. To test this hypothesis, we introduced 3-year-old children to the same typical category instances used in 
the Instance condition, this time labeling them with a novel (Japanese) superordinate term, for which the children had no prior associates. These foreign terms allowed us to observe the effect of labeling, per se, without contaminating the results with established associations between English superordinate terms and specific category members. Like the Group and Instance conditions, success in the Japanese Label condition required that children generate a superordinate category from a set of instances, and go on to select several appropriate category members. If coupling the set of instances with the unfamiliar Japanese label highlights the superordinate relations among the materials, then performance in the Japanese Label condition should exceed performance in the Instance condition. If, on the other hand, labeling plays no such role, then performance in the Japanese Label and Instance conditions should be indistinguishable.

\section{Method}

Twelve 3-year-old children (mean age $=3.7$ ), drawn from the same population as the previous experiment, served as subjects. The stimuli and the familiarization period were identical to those described in Experiment I. In the experimental session, the experimenter told children that the puppet came from another planet and could not speak English, but said, for example, that he wanted dobutsus. She then said, "I don't know what dobutsus means, but I know he likes things like a dog, or a duck, or a horse." She showed the child drawings of these items as she mentioned them and then asked the child to help her find other things the puppet might want. This procedure was continued, using the Japanese superordinate terms gohans and kimonos for food and clothes. Order of category presentation was counterbalanced.

\section{Results and Discussion}

Children's performance in the Japanese Label condition (Experiment II) was compared to that of their age-mates in the Label and Instance conditions (Experiment I). When the instances were presented in conjunction with the Japanese labels, 3-year-olds formed superordinate classes successfully (mean score $=$ 10.53) (see Figure 2). Despite the fact that the Japanese words had no prior meaning for the children, their performance in the Japanese Label condition (Experiment II) was significantly better than their age-mates' performance in the Instance condition (Experiment I), one-tailed $t(44)=4.41, p<.02$. There was no significant difference between 3-year-olds' performance in the Label (Experiment I) and Japanese Label (Experiment II) conditions, $t(44)=.78, p<.38$. The effect of the novel labels was striking: The Japanese label effectively highlighted the taxonomic relations and licensed the induction of the superordinate categories.

One description of this finding is that the children were implicitly 'translating' the Japanese label (e.g., dobutsu) into a known English label (e.g., animal). In 


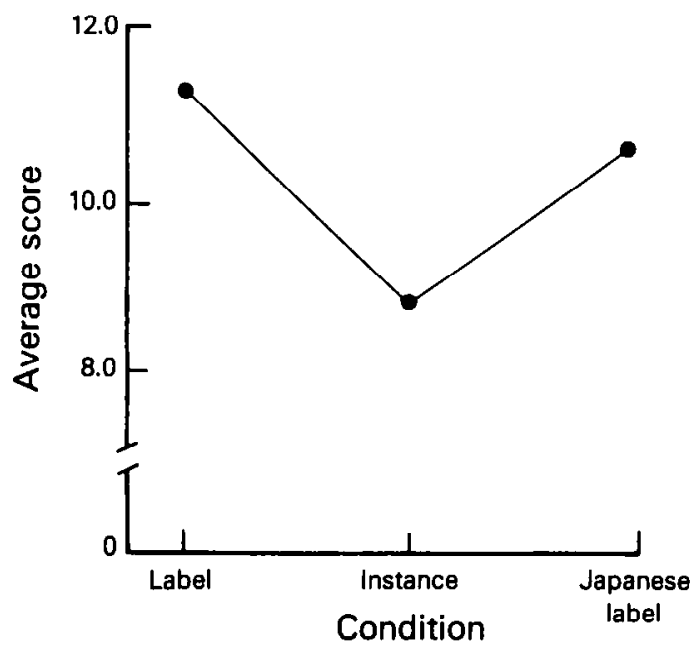

Figure 2. Mean scores for 3-year-old children in the Label, Instance (Experiment I), and Japanese Label (Experiment II) Clue conditions

fact, there is anecdotal evidence suggesting that this was true for at least some of our 3-year-olds. Several of the children in the Japanese Label condition told us, for example, "Oh! That puppet wants ANIMALS!" as they sorted the drawings. These children, unlike those in the Instance condition (Experiment I), successfully generalized from the instances to higher order superordinate-level categories.

This illustrates a very important point. Since they knew no Japanese, they could only have arrived at these superordinate labels from the instances. Yet as we have seen (Experiment I, Instance condition), 3-year-olds have some difficulty generating superordinate categories from instances alone. Additionally, when 3-year-olds are presented with a set of drawings (e.g., cow, horse, duck, fish) from a single superordinate category and are asked explicitly to indicate their common class label (e.g., animal), they have difficulty doing so. Although they are familiar with these superordinate labels, young children's overwhelming tendency is to name each individual drawing (Waxman, 1985). Clearly, the superordinate relations among sets of instances are relatively inaccessible to 3year-old children when no common label is presented.

In sum, the introduction of the Japanese label dramatically altered children's access to superordinate relations. The single label, albeit a foreign one, served as a powerful taxonomic clue, drawing the different members together to form a single cohesive category. This finding lends strong support to the hypothesis that taxonomic relations become especially salient in word learning. The bias to interpret novel labels taxonomically is available to children as young as $\mathbf{3}$ years of age, and is particularly useful at superordinate levels. 


\section{GENERAL DISCUSSION}

We have addressed two possible explanations for the discrepancy between young children's access to superordinate relations in classifcation and word learning. First, we tested the hypothesis that certain aspects of classification tasks may obscure children's appreciation of superordinate relations. Specifically, we argued that in free classification, the open-ended instructions leave the choice among possible organizational schemes unconstrained, allowing for virtually any grouping of the stimuli (e.g., idiosyncratic, thematic), not necessarily a taxonomic one. The results of Experiment I are consistent with this hypothesis: Using clues to focus children's attention on taxonomic relations allowed them to demonstrate their appreciation of superordinate relations.

Second, we addressed the hypothesis that the ease with which children access superordinate relations in the context of word learning reflects something special about language. Specfically, we argued that if children honor an implicit bias to interpret novel nouns as referring to taxonomic relations, this bias should be evident early in development, particularly at superordinate levels where children find taxonomic relations difficult. By using several different types of clues, we were able to compare the effect of the Japanese label with that of other clues (including the English superordinate label, a set of typical category instances and explicit instructions to form a group). Empirical support for our second hypothesis came from the difference between 3-year-olds' classification with, and without, a label. Without labels, 3-year-old children had difficulty inducing inclusive superordinate-level categories from a set of typical category members. The introduction of a single (yet foreign) label effectively alerted children to the taxonomic relations among these instances and licensed the induction of superordinate categories. Simply introducing an unfamiliar label in conjunction with the instances led children to classify as successfully as their age-mates in the English Label condition.

We used classification tasks in these experiments because we wanted robust tests of young children's ability to establish and maintain superordinate-level classification schemes. Since childten in the Japanese Label condition readily induced superordinate categories from the instances and used that taxonomic relation consistently as a guide in classification, we have unambiguous evidence that children are sensitive to a powerful taxonomic constraint in a word learning.

Although this bias to use labels to refer specifically to taxonomic relations is evident in very young children, it is not unique to children of a particular developmental stage. Throughout development, we tend to lexicalize taxonomic relations (e.g., food), but describe thematic (e.g., a knife and an apple) and ad hoc (e.g., items useful in French cooking) relations with phrases and sentences. This linguistic convention is common across languages, both spoken (Berlin, 1978) and signed (Newport \& Bellugi, 1978) and reflects a bias on the part of speakers to interpret single nouns as labels for taxonomic classes. Very young 
children are sensitive to this bias, using single nouns to highlight superordinate relations.

Finally, let us return to a question raised earlier in this paper: Why might children need clues to focus on superordinate relations in classification but not in interpreting word meaning? As we mentioned earlier, there are many possible ways to classify objects. This flexibility in classification affords us cognitive power and creativity. However, if unconstrained, this flexibility could greatly complicate the task of word learning. Given the myriad possible relations among objects (e.g., thematic, idiosyncratic), how would young children learn that labels usually refer to taxonomic relations and rule out other possible relations? To make word learning possible, we may need constraints on that flexibility. In fact, we may need these constraints most at superordinate levels where taxonomic relations are less readily accessible.

The results of our experiments provide evidence of just this sort of constraint. Children as young as 3 years of age interpret novel labels taxonomically and find them especially useful in making an induction from instances to superordinate categories. Their performance provides support for both our hypotheses regarding the flexibility of classification and a powerful constraint in word learning. These findings lead us to the conclusion that classification and word learning are intimately linked, each serving to guide and reinforce the young child's appreciation of taxonomic relations.

\section{REFERENCES}

Anglin, J.M. (1977). Word, object and concept development. New York: W.W. Norton.

Anglin, J.M. (1979). The child's first terms of reference. In N.R. Smith \& M.B. Franklin (Eds.), Symbolic functioning in childhood. Hillsdale, NJ: Erlbaum.

Berlin, B. (1978). Ethnobiological classification. In E.H. Rosch \& B. B. Lloyd (Eds.), Cognition and categorization. Hillsdale, NJ: Erlbaum.

Brown, R. (1957). Linguistic determinism and the part of speech. Journal of abnormal and social psychology. 55, 1-5.

Callanan, M.A. (1982). Parental input and young children's acquisition of hierarchially organized concepts. Unpublished doctoral dissertation, Stanford University, Palo Alto, CA.

Carey, S. (1978). The child as a word-learner. In M. Halle, J. Bresnan, \& G.A. Miller (Eds.), Linguistic theory and psychological reality. Cambridge, MA: MIT Press.

Clark, E.V. (1983). Meanings and concepts. In J.H. Flavell \& E.M. Markman (Eds.), Handbook of child psychology: Vol. 3. Cognitive development. New York: Wiley.

Gelman, R., \& Baillargeon, R. (1983). A review of some Piagetian concepts. In J.H. Flavell \& E.M. Markman (Eds.), Handbook of child psychology: Vol. 3. Cognitive development. New York: Wiley.

Gelman, S.A., \& Taylor, M. (1984). How two-year-old children interpret common and proper names for unfamiliar objects. Child Development, 51, 1535-1540.

Horton, M.S., \& Markman, E.M. (1980). Developmental differences in the acquisition of basic and superordinate categories. Child Development, 51, 708-719.

Inhelder, B., \& Piaget, J. (1964). The early growth of logic in the child. New York: W.W. Norton.

Katz, N., Baker, E., \& MacNamara, J. (1974). What's in a name? A study of how children learn common and proper names. Child Development, 4, 469-473. 
Keil, F.C. (1979). Semantic and conceptual development. Cambridge, MA: Harvard University Press.

Kendler, H.H., \& Guenther, K. (1980). Developmental changes in classificatory behavior. Child Development, 51, 339-348.

Kobasigawa, A., \& Middleton, D.B. (1972). Free recall of categorized items by children at three grade levels. Child Development, 43, 1067-1072.

Kripke, S. (1972), Naming and necessity. In D. Davidson \& G. Harmon (Eds.), Semantics and natural language. Dordrecht, Netherlands: Reidl.

Landau, B., \& Gleitman, L.R. (1985). The language of perception in a blind child. Cambridge, MA: Harvard University Press.

MacNamara, J. (1982). Names for things: A study of human learning. Cambridge, MA: Harvard University Press.

Mandler, J.M. (1983). Representation. In J.H. Flavell \& E.M. Markman (Eds.), Handbook of child psychology: Vol. 3. Cognitive development. New York: Wiley.

Markman, E.M. . Cox, B., \& Machida, S. (1981). The standard object-sorting task as a measure of conceptual organization. Developmental Psychology, 17, 115-117.

Markman, E.M., \& Callanan, M.A. (1983). An analysis of hierarchical classification. In R. Stemberg (Ed.), Advances in the psychology of human intelligence. Hillsdale, $\mathrm{NJ}$ : Erlbaum.

Markman, E.M., \& Hutchinson, J.E. (1984). Children's sensitivity to constraints on word meaning: Taxonomic vs. thematic relations. Cognitive Psychology, 16, 1-27.

Mervis, C.B., \& Pani, J.R. (1980). Acquisition of basic object categories. Cognitive Psychology, $12,496-522$.

Nelson, K. (1978). How children represent knowledge of their world in and out of language: A preliminary report. In R.S. Siegler (Ed.), Children's thinking: What develops? Hillsdale, NJ: Erlbaum.

Newport, E.L., \& Bellugi, U. (1978). Linguistic expression of category levels in a visual-gestural language: A flower is a flower is a flower. In E.H. Rosch \& B.B. Lloyd (Eds.), Cognition and categorization. Hillsdale, NJ: Erlbaum.

Quine, W.V.O. (1960). Word and object. Cambridge, MA: MIT Press.

Rescorla, L.A. (1981). Category development in early language. Journal of child language, 10, 571-588.

Rosch, E., Mervis, C.B., Gay, W.D., Boyes-Braem, P., \& Johnson, D.N. (1976). Basic objects in natural categories. Cognitive Psychology, 8, 382-439.

Shipley, E.F., Kuhn, I.F., \& Madden, C. (1983). Mothers' use of superordinate category terms. Journal of Child Language, 10, 571-588.

Smiley, S.S., \& Brown, A.L. (1979). Conceptual preference for thematic or taxonomic relations: A nonmonotonic trend from preschool to old age. Journal of Experimental Child Psychology, 28, 249-257.

Smith, E.E., \& Medin, D.L. (1981). Categories and concepts. Cambridge, MA: Harvard University Press.

Snodgrass, J.G., \& Vanderwart, M. (1980). A standardized set of 260 pictures: Norms for name agreement, image agreement, familiarity, and visual complexity. Journal of Experimental Psychology: Human Learning and Memory, 6(2), 174-215.

Sugarman, S. (1983). Children's early thought: Developments in classification. Cambridge, MA: Harvard University Press.

Waxman, S.R. (1983). Superordinate classification in preschool children. Paper presented at the meeting of the Society for Research in Child Development, Detroit, MI.

Waxman, S.R. (1985). Hierarchies in classification and language: Evidence from preschool children. Unpublished doctoral dissertation, University of Pennsylvania, Philadelphia. 